OPEN ACCESS

Edited by:

Akos T. Kovacs,

University of Jena, Germany

Reviewed by:

David Baltrus,

University of Arizona, USA

Claudio Valverde,

National University of Quilmes,

Argentina

César De La Fuente,

Massachusetts Institute

of Technology, USA

*Correspondence:

Eric Déziel

eric.deziel@iaf.inrs.ca

Specialty section:

This article was submitted to

Microbial Physiology and Metabolism,

a section of the journal

Frontiers in Microbiology

Received: 05 July 2016 Accepted: 23 December 2016

Published: 10 January 2017

Citation:

Jean-Pierre F, Tremblay $J$ and

Déziel E (2017) Broth versus

Surface-Grown Cells: Differential

Regulation of RsmY/Z Small RNAs

in Pseudomonas aeruginosa by

the Gac/HptB System.

Front. Microbiol. 7:2168

doi: 10.3389/fmicb.2016.02168

\section{Broth versus Surface-Grown Cells: Differential Regulation of RsmY/Z Small RNAs in Pseudomonas aeruginosa by the Gac/HptB System}

\author{
Fabrice Jean-Pierre ${ }^{1}$, Julien Tremblay ${ }^{1,2}$ and Eric Déziel ${ }^{1 *}$ \\ ${ }^{1}$ Institut National de la Recherche Scientifique, Institut Armand-Frappier, Laval, QC, Canada, ${ }^{2}$ National Research Council \\ Canada, Montréal, QC, Canada
}

Two-component systems are capable of profoundly affecting genetic regulation in bacteria by detecting environmental stimuli, allowing them to quickly adapt. In Pseudomonas aeruginosa, the small RNAs (sRNAs) RsmY and RsmZ are under the control of the GacS/A system. They have been described as ones of the major key players in the control of planktonic and surface-associated behaviors. Genetic regulation by these sRNAs is achieved by the titration of the negative post-transcriptional regulator RsmA which affects the expression of over 500 genes. There is increasing evidence pinpointing the importance of RsmY and RsmZ in the planktonic-sessile $P$. aeruginosa lifestyles switch control. Using swarming motility as a model, we show here that these sRNA are differentially regulated depending on the selected growth conditions (i.e., planktonic versus surface grown-cells). Also, we report that opposite to planktonically grown cells, rsm $Z$ regulation does not implicate the response regulator GacA in swarming cells. Furthermore, we present data indicating that RsmY/Z expression influence swarming motility via the protein $\mathrm{HptB}$ which acts as a negative regulator of these sRNAs and that they do not strictly converge to RsmA as previously reported.

Keywords: swarming, broth-surface, small RNAs, surface motility, genetic regulation, histidine phosphotransfer protein, HptB

\section{INTRODUCTION}

Bacterial survival in the environment relies on their capacity to quickly adapt to changing conditions by either inducing or repressing specific sets of genes (Boor, 2006). Some bacteria have the ability to colonize a broad range of hosts using virulence functions (Furukawa et al., 2006). One adaptation mechanism used by bacteria consist of two-component systems (TCSs) (Hoch and Varughese, 2001), membrane-bound sensors coupled to cytoplasmic response regulators that permit the integration of external stimuli and induce global gene expression shifts (Beier and Gross, 2006). The heterotrophic opportunistic pathogen Pseudomonas aeruginosa exemplifies such a remarkable capacity to adapt to changing environments by encoding more than 60 TCS on its genome (Rodrigue et al., 2000). The GacS/GacA TCS has been extensively described over the years and is central in regulating the expression of the two small RNAs (sRNAs) RsmY and RsmZ. Mainly controlled by the response regulator GacA, these sRNAs titrate the availability of RsmA, a post-transcriptional regulator modulating the expression of functions implicated in the transition 
between the surface-associated and the planktonic $P$. aeruginosa lifestyles (Hoch and Varughese, 2001; Brencic and Lory, 2009; Brencic et al., 2009).

Swarming motility is a surface-associated type of bacterial motility characterized by a rapid and coordinated movement of a bacterial population on a viscous surface (Kearns, 2010). In $P$. aeruginosa, this type of bacterial movement necessitates the presence of functional flagella and the production of rhamnolipids (and other RhlABC products) responsible for both modulation of the surface behavior and lowering surface tension (Déziel et al., 2003; Tremblay et al., 2007). Over the years, swarming motility has been linked to many phenotypes, such as increased antibiotic resistance, and to be inversely regulated to another surface bacterial behavior, biofilm formation (Caiazza et al., 2007; Lai et al., 2009). Given that swarming motility represents a distinct bacterial lifestyle from planktonic and sessile cells, many studies have aimed at understanding how this surface behavior is regulated. Over the years, the secondary messenger molecule, c-di-GMP has been shown to have a profound impact over the motile-sessile behavior in the bacterial kingdom, but in $P$. aeruginosa its implication in swarming motility is still not completely clear yet and is a matter of active research as some contradictions have been reported (Caiazza et al., 2007; Kuchma et al., 2007; Merritt et al., 2007; Baker et al., 2016). Excluding the impact of secondary messengers, this surface behavior is still poorly understood at the expression level. In an attempt to better characterize swarming motility, Overhage et al. (2008), defined how this surface behavior was affected and observed that many virulence genes were upregulated in swarming cells compared to their planktonic (free-swimming) counterpart. To further dissect and understand the complexity of swarming motility, we have previously (Tremblay and Déziel, 2010) conducted a transcriptomic analysis of swarming cells compared to bacteria that were grown on the same agar-solidifed medium just dried longer to prevent swarming motility (surface versus surface). In contrast with Overhage et al. (2008), we found that cells at the migrating tip of a swarming colony downregulated virulence factor expression but upregulated genes associated with energy metabolism. The apparent discrepancies between the two studies were explained by differences in experimental design. Indeed, Overhage et al. (2008), compared cells at the swarm tip against planktonic cells (surface versus broth) whereas Tremblay and Déziel (2010) compared surface versus surface. The observation of such opposite results between these two studies also raised the possibility of the existence of unknown regulation pathways specific to surface-grown cells, here in the context of swarming motility.

The study of swarming motility at the regulatory level is complex given that two critical factors, namely flagellar function and biosurfactants production, are imperative for that surface behavior. Thus, to better understand how swarming motility is regulated, it is helpful to identify mutants incapable of such a type of social movement while still possessing a functional flagellum and with no defect in production of rhamnolipids. Interestingly, a mutation in the $h p t B$ gene, encoding for one of the three histidine phosphotransfer proteins implicated in signal transduction in $P$. aeruginosa, was reported to negatively affect swarming motility (Hsu et al., 2008). A microarray analysis of a $\triangle h p t B$ mutant has shown that many flagellarrelated genes are affected but surprisingly, no defect in swimming motility was observed (Bhuwan et al., 2012). Also, the production of rhamnolipids was never addressed in these studies, thus not covering all the possibilities as to why such a mutant is incapable of swarming motility. Furthermore, HptB was reported to interact with hybrid sensor kinases, one of which is RetS implicated in the control of the GacS/GacA system to affect global gene expression (Goodman et al., 2004; Lin et al., 2006; Brencic et al., 2009; Bordi et al., 2010). Interestingly, Bordi et al. (2010) determined that HptB mediates its effect through the modulation of the sRNA RsmY exclusively.

In the present study, we demonstrate how HptB affects swarming motility via modulation of the expression of both RsmY and RsmZ and that the swarming default of an $\Delta h p t B$ mutant is not associated with flagellar malfunction nor insufficient biosurfactant production. Comparing $\Delta h p t B$ mutant cells cultivated in planktonic versus surface conditions, we show that both $\operatorname{rsm} Y$ and $r \sin Z$ are negatively regulated by $\mathrm{HptB}$. Furthermore, HptB-mediated $r s m Z$ expression control is stronger in swarming cells and, unexpectedly, not under the absolute control of the GacA response regulator.

\section{MATERIALS AND METHODS}

\section{Strains, Plasmids, and Growth Conditions}

Bacteria used in this study are all derived from the parental strain PA14 (Rahme et al., 1995; Lee et al., 2006) and are listed in Table 1. Bacteria were cultivated at $37^{\circ} \mathrm{C}$ in Tryptic Soy Broth (TSB) (Difco) with shaking (240 rpm) in a TC-7 roller drum (New Brunswick) or on TSB plates solidified with $1.5 \%$ agar. For swarming motility assays, overnight cultures were washed in phosphate-buffered saline (PBS) and diluted to the desired $\mathrm{OD}_{600}$. For transcriptomic analyses, the bacteria were cultivated in M9 minimal medium supplemented with $11 \mathrm{mM}$ dextrose and $0.5 \%$ casamino acids (Difco) (M9DCAA) broth at $34^{\circ} \mathrm{C}$ (Tremblay and Déziel, 2008). $\mathrm{OD}_{600}$ was measured with a Nanodrop ND1000 (Thermo Fisher Scientific) and pathlength correction was applied by multiplying the given value by a factor of 10 .

\section{Construction of the $\Delta h p t B$ Knock-Out Mutant}

Deletion of the Hpt domain of the $h p t B$ gene (PA3345 in PAO1 and PA14_20800 in PA14) was performed as follows. Two fragments of DNA were amplified from PA14 genomic DNA using pairs of primers PA3345_Left_FWD and PA3345_ Left_REV for the left fragment and PA3345_Right_FWD and PA3345_Right_REV for the right fragment. These fragments 
TABLE 1 | Strains/plasmids used in this study.

\begin{tabular}{|c|c|c|c|}
\hline Strains/plasmids & ED \# & Phenotype/genotype & Reference \\
\hline \multicolumn{4}{|l|}{ E. coli } \\
\hline $\mathrm{DH} 5 \alpha$ & 78 & fhuA2 $\Delta$ (argF-lacZ)U169 phoA glnV44 Ф80 $\Delta$ (lacZ)M15 gyrA96 recA1 relA1 endA1 thi-1 hsdR17 & Woodcock et al., 1989 \\
\hline SM10 ( $\lambda$ pir) & 222 & thi thr leu tonA lacY supE recA::RP4-2-Tc::Mu Km $\lambda$ pir & Simon et al., 1983 \\
\hline \multicolumn{4}{|l|}{$P$. aeruginosa } \\
\hline PA14 & 14 & UCBPP-PA14 wild-type strain & Rahme et al., 1995 \\
\hline$\Delta h p t B$ & 1214 & Markerless deletion of $h p t B$ in PA14 & This study \\
\hline$\Delta r s m Y$ & 1971 & Markerless deletion of $r s m Y$ in PA14 & Laboratory collection \\
\hline$\Delta r s m Z$ & 1976 & Markerless deletion of rsmZ in PA14 & Laboratory collection \\
\hline$\Delta r s m Y Z$ & 1998 & Markerless deletion of rsmY and rsmZ in PA14 & Laboratory collection \\
\hline $\operatorname{gacA}^{-}$ & 1800 & MrT7 transposon mutant ID $34781 \mathrm{Gm}^{\mathrm{R}}$ & Liberati et al., 2006 \\
\hline$\Delta h p t B g a c A$ & 2654 & Markerless hptB deletion in PA14, gacA::MrT7 transposon mutant ID $34781 \mathrm{Gm}^{\mathrm{R}}$ & This study \\
\hline$\Delta h p t B r s m Y$ & 2831 & Markerless deletion of $h p t B$ and $r s m Y$ in PA14 & This study \\
\hline$\Delta h p t B r s m Z$ & 2832 & Markerless deletion of $h p t B$ and rsmZ in PA14 & This study \\
\hline$\Delta h p t B r s m Y Z$ & 2833 & Markerless deletion of $h p t B, r s m Y$ and $r s m Z$ in PA14 & This study \\
\hline \multicolumn{4}{|l|}{ Plasmids } \\
\hline pEX18Tc & & Suicide vector in $P$. aeruginosa $\operatorname{SacB}, \mathrm{Tc}^{\mathrm{R}}$ & Hoang et al., 1998 \\
\hline pJT39 & 1306 & Suicide vector containing the hptB gene with an absent histidine phosphotransfer domain & This study \\
\hline pEXG2- $\Delta r s m Y$ & & Suicide vector for $\Delta r s m Y$ mutation construction & Brencic et al., 2009 \\
\hline pEXG2- $\Delta r s m Z$ & & Suicide vector for $\Delta r s m Z$ mutation construction & Brencic et al., 2009 \\
\hline
\end{tabular}

were ligated together using a complementary engineered overhang sequences of 15 nucleotides common to PA3345 _Left_REV and PA3345_Right_FWD. The DNA fragment was then cloned in the MCS of pEX18Tc using restriction enzymes HindIII and SmaI. The resulting plasmid (pEX18Tc_ $\Delta h p t B$ ) was transformed into Escherichia coli SM10 on TSB plates containing $15 \mu \mathrm{g} \mathrm{ml}^{-1}$ of tetracycline (Tc). A two partner conjugation between PA14 and SM10-pEX18Tc_ $\Delta h p t B$ was then performed. Tetracycline resistant simple recombinants were selected on tetracycline $125 \mu \mathrm{g} \mathrm{ml}^{-1}$ TSB plates and streaked onto TSB without antibiotic overnight. Double recombinants were selected by plating these overnight grown cells on $\mathrm{LB}$ (without $\mathrm{NaCl}$ ) supplemented with $8 \%$ sucrose. Positive clones were confirmed by polymerase chain reaction (PCR) and sequencing.

\section{Motility Assays}

Swarming motility assays were performed using the same medium as for swimming motility tests but with $0.5 \%$ agar. Once autoclaved, the semi-solid agar was dried in a laminar biological safety cabinet for $75 \mathrm{~min}$. Swarming plates were inoculated with $5 \mu \mathrm{l}$ of a bacterial subculture grown in TSB to early stationary phase and adjusted to an $\mathrm{OD}_{600}$ of 3.0 in sterile $1 \mathrm{X}$ PBS. The plate were then incubated overnight at $34^{\circ} \mathrm{C}$ (Tremblay and Déziel, 2008). Time-lapse image analysis was done using Photoshop CS3 Extended (Adobe).

Swimming motility plates were done by inoculating $3 \mu \mathrm{l}$ of bacterial suspension at an $\mathrm{OD}_{600}$ of 3.0 directly inside $\mathrm{LB}$ or M9DCAA plates solidified with $0.25 \%$ agar. The swimming diameter was measured after overnight incubation at $34^{\circ} \mathrm{C}$. Experiments for testing swimming and swarming motility were performed with a minimum of three to five technical replicates on two separate days. Statistical analysis was done using Prism version 6.0 (GraphPad).

\section{RNA Preparation}

Total RNA was extracted from liquid bacterial cultures grown in triplicate in M9DCAA cultivated to late exponential phase $\left(\mathrm{OD}_{600}=1.3\right)$ at $34^{\circ} \mathrm{C}$. The cells were then centrifuged for $5 \mathrm{~min}$ at $12,000 \times g$ and the supernatant was discarded. Cells were resuspended in PureZOL (BioRad) and RNA extraction was performed following the manufacturer's recommendations. For surface-grown bacteria, RNA was collected from the cells located at the migrating tip of a swarming colony grown for $12 \mathrm{~h}$ at $34^{\circ} \mathrm{C}$ using $8 \mu \mathrm{l}$ of RNAlater (Qiagen) that was put directly on each tendril tip, as previously described (Tremblay and Déziel, 2010). Cells were resuspended by pipetting and transferred to a $1.5 \mathrm{ml}$ microcentrifuge tube kept on ice. An average of 8 migrating tendril tips were harvested per plate with three plates for each biological replicate for a total of nine plates. RNA extraction was performed using PureZOL.

\section{Quantitative Real-Time PCR (qRT-PCR)}

Quantitative real-time PCR (qRT-PCR) was done with the $q$ Script ${ }^{\mathrm{TM}}$ One-Step SYBR Green kit and a RotorGene 6000 (Corbett) thermocycler. Primers were designed in order to obtain amplicons of $80-150 \mathrm{bp}^{1}$. The $\operatorname{nadB}$ gene was used as control. Each cycle of qRT-PCR was done in triplicate. The threshold cycle $(C t)$ was normalized to $\operatorname{nadB} C t$ amplified in each corresponding samples. Variation in expression was calculated using the $-2^{\Delta \Delta C t}$ method (Livak and Schmittgen, 2001). Assessment of variation in expression was performed using biological triplicates on two different days. Statistical analysis was done using Prism version 6.0 (GraphPad). Gene expression variation is shown as Relative expression variation $\left(\log _{2}\right)$ to the wild-type PA14 strain.

\footnotetext{
${ }^{1}$ http://frodo.wi.mit.edu/primer3/
} 


\section{LC/MS Rhamnolipids Quantification}

Concentrations of rhamnolipids were determined by liquid chromatography(LC)/mass spectrometry (MS) (Abdel-Mawgoud et al., 2014). A $400 \mu \mathrm{l}$ sample of a liquid overnight culture was collected and the $\mathrm{OD}_{600}$ was measured. Then the samples were centrifuged at $16,000 \times g$ for $10 \mathrm{~min}$. to remove bacterial cells. To $300 \mu \mathrm{l}$ of supernatant, $300 \mu \mathrm{l}$ acetonitrile containing $20 \mathrm{mg} / \mathrm{L}$ 5,6,7,8-tetradeutero-4-hydroxy-2-heptylquinoline (HHQ-d4) as the internal standard were added. Samples were analyzed by high-performance liquid chromatography (HPLC; Waters 2795, Mississauga, ON, Canada) equipped with a C8 reversephase column (Kinetex, Phenomenex) using a water/acetonitrile gradient with a constant $2 \mathrm{mmoll}^{-1}$ concentration of ammonium acetate. The detector was a mass spectrometer (Quattro Premier $\mathrm{XE}$, Waters). Analyses were carried out in the negative electrospray ionization (ESI-) mode.

Surface biosurfactant production was measured by inoculating the $\Delta h p t B$ mutant and the wild-type strain on swarm plates. After overnight incubation at $34^{\circ} \mathrm{C}$, all the agar was recovered from the Petri dishes and transferred to an Erlenmeyer flask containing 1:1 (v/v) $\mathrm{KHCO}_{3}(\mathrm{pH}$ 9.0) (i.e., for five $20 \mathrm{ml}$ swarm plates, $100 \mathrm{ml}$ of $\mathrm{KHCO}_{3}$ was added). The collected agar was vigorously mixed for $1 \mathrm{~h}$ at room temperature then filtered through Whatman no. 1 covered with silica sand (Fisher). The filtrate was acidified at $\mathrm{pH} 4.0$ with concentrated $\mathrm{HCl}$ then extracted three times with $50 \mathrm{ml}$ ethyl acetate. The organic phases were pooled, evaporated and the residue analyzed as described above. Total biomass was quantified by resuspending bacteria on the swarm plates twice with $1 \mathrm{ml}$ PBS then transferred to preweighed aluminum boats, dried at $65^{\circ} \mathrm{C}$ for $2 \mathrm{~h}$ and then weighed again. Biomass was measured by preparing a parallel swarm set in identical conditions for rhamnolipids quantification. Rhamnolipids quantification was done in technical triplicate on two different days. Statistical analysis was done using Prism version 6.0 (GraphPad).

\section{RESULTS}

\section{HptB-Mediated Swarming Regulation Does Not Implicate Flagellar Malfunction nor a Default in Rhamnolipids Production}

There is increasing evidence for the implication of the HptB phosphotransfer protein in the modulation of several P. aeruginosa behaviors (Hsu et al., 2008; Bordi et al., 2010; Kong et al., 2013). Recently, swarming motility has been identified as being under the control of the $\mathrm{HptB}$ regulon, which was determined to include flagella-related genes (Bhuwan et al., 2012); however, surprisingly, no effect was seen on flagellar functionality. To further characterize the role of $\mathrm{HptB}$ in the regulation of the swarming surface-associated behavior, we engineered a markerless $\Delta h p t B$ mutant by deleting the entire histidine phosphotransfer domain (Hsu et al., 2008). As previously noted by Hsu et al. (2008), for strain PAO1, inactivation of the $h p t B$ gene in strain PA14 results in the loss of swarming motility (Figure 1A). A functional flagellum and the production of RhlABC products (mostly rhamnolipids) are elements required for swarming motility (Köhler et al., 2000; Déziel et al., 2003; Caiazza et al., 2005; Tremblay et al., 2007). Thus, we verified whether or not flagellar functionality is responsible for the $\triangle h p t B$ mutant swarming phenotype by performing swimming motility assays. As shown in Figure 1B, the $\Delta h p t B$ mutant still has a functional flagellum in both tested conditions. The production of rhamnolipids was also verified both in planktonic cultures and directly on swarm plates. The $\Delta h p t B$ mutant produces an equivalent amount of biosurfactant compared to the wild-type strain when incubated in M9DCAA broth (Figure 1C). When looking at production of rhamnolipids by swarming cells directly from plates the $\Delta h p t B$ mutant is not impaired and it actually displays increased biosurfactant production (Figure 1D). Absence of a growth defect by the $\Delta h p t B$ mutant (Supplementary Figure S1) excludes known factors for the observed deficient swarming phenotype. We conclude that $\mathrm{HptB}$ positive control on swarming motility does not go through the known required factors.

\section{Swarming Motility is Linked to sRNAs Expression}

There are indications that expression of the swarming phenotype is positively regulated by RsmA and antagonized by the small RNAs, RsmY and RsmZ (Heurlier et al., 2004; Kay et al., 2006). In cells cultivated as broth cultures, $\mathrm{HptB}$ negatively affects the expression of RsmY specifically, but with no effect on RsmZ (Bordi et al., 2010). Since bacterial growth mode (i.e., cells grown planktonically versus on a surface) can affect the output of genetic regulation (Overhage et al., 2008; Tremblay and Déziel, 2010), we monitored the expression of both $r s m Y$ and $r s m Z$ in bacteria grown in both planktonic and swarming cultures. In agreement with what was seen in strain PAK (Bordi et al., 2010), the RsmY sRNA is also overexpressed in the PA14 $\Delta h p t B$ mutant grown in broth cultures while a small (and not previously reported) increase in expression of RsmZ is also observed (Figure 2A). Surprisingly, when the same experiment is carried out on swarming cells sampled at the migrating tip of a swarming colony, a marked increase of $2 \log _{2}$ in $r s m Z$ expression is observed when compared to its planktonic counterpart (Figure 2A), suggesting specific upregulation by surface growth. We therefore looked at the swarming phenotypes of $\Delta r s m Y, \Delta r s m Z$, and $\Delta r s m Y Z$ mutants and observed that they all exhibit a hyperswarming phenotype (Figure 2B), when compared to the wild-type (Figure 1A). To precisely confirm this hyperswarmer behavior, we measured the area covered by the swarming colony using time-lapse image analysis: $\Delta r s m Y$ and $\triangle r s m Z$ behave the same way while the increased swarming phenotype of the double mutant is even more pronounced than both simple mutants (Figure 2C; Supplementary Figure S2).

To verify the hypothesis that the swarming defect of the $\Delta h p t B$ mutant is directly caused by the overexpression of $r s m Y$ and $r s m Z$, we constructed double $\Delta h p t B r s m Y, \Delta h p t B r s m Z$ and triple $\Delta h p t B r s m Y Z$ mutants. Significantly, the introduction of either a $r s m Y$ or a $r s m Z$ mutation in a $\Delta h p t B$ background resulted in rescue of the swarming phenotype, yet not identical as the 
A

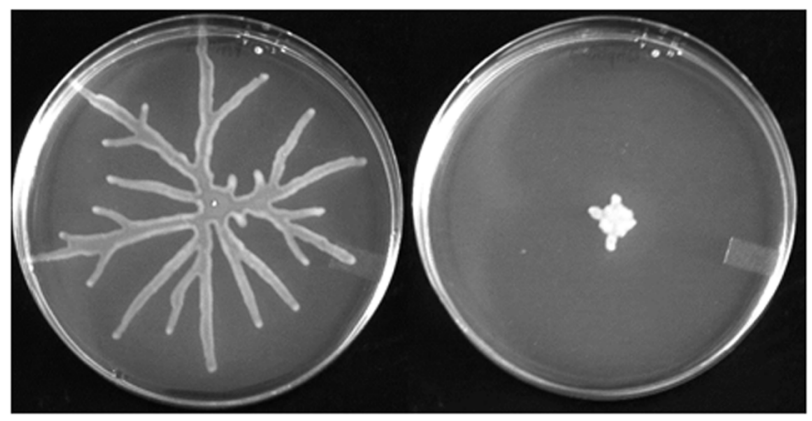

PA14

$\Delta h p t B$

\section{B}

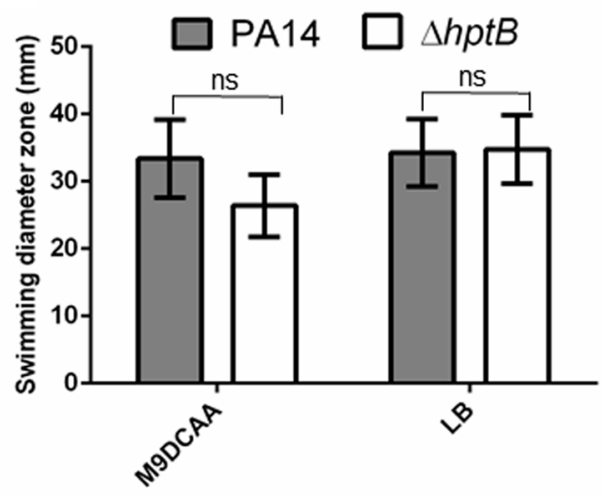

C

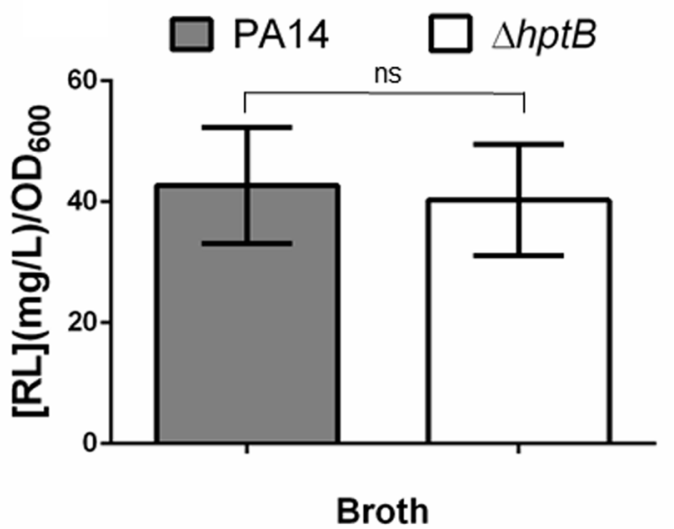

D

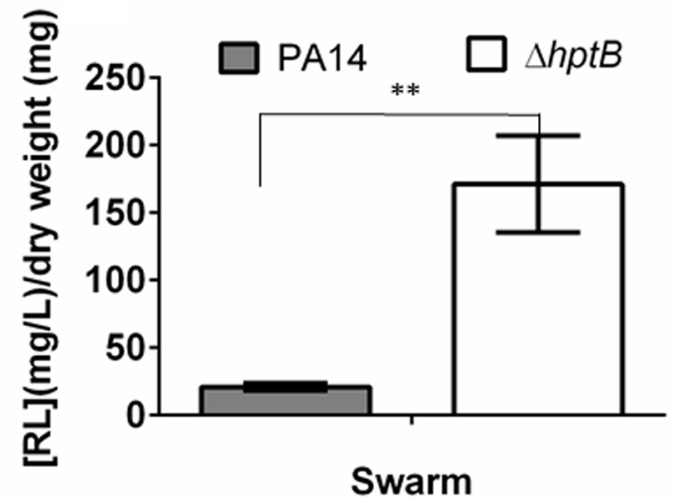

FIGURE 1 | A mutation in the $h p t B$ gene affects swarming motility. (A) Swarming motility phenotypes of the wild-type PA14 strain and the $\Delta h p t B$ mutant. (B) Swimming motility assay in various media containing $0.25 \%$ agar. (C) Rhamnolipids quantification in planktonic cultures. (D) Rhamnolipids quantification on swarming plates. Error bars represent the standard error of the mean for experiments carried out at least twice with a minimum of three replicates per experiment. Student's $t$-test analysis was applied on two independent experiments with ${ }^{* *} p<0.01$; ns, not significant.

pattern observed for the simple $\Delta r s m Y$ and $\Delta r s m Z$ mutants (Figure 3A). Finally, swarming motility of the triple $\triangle h p t B r s m Y Z$ exhibited exactly the same phenotype as the double $\triangle r s m Y Z$ (Figure 3B). Taken together, these results support a model where HptB promotes swarming motility through the RsmA pathway, likely by decreasing the expression of both inhibitory sRNAs RsmY and RsmZ.

\section{Regulation of rsmZ Differs in Surface-Grown Cells Compared to their Planktonic Counterpart}

To understand if the regulation of $r s m Y$ and $r s m Z$ by HptB is exerted through the GacS/GacA TCS, which has been characterized as being responsible for the transcription of these two sRNAs in liquid cultures (Brencic et al., 2009), we engineered a double $\triangle h p t B g a c A$ mutant (Supplementary Material). In that mutant background, we monitored the expression of $r s m Y$ and $r s m Z$ in planktonic and surfaceassociated (swarming) cells. When the cells are cultivated in broth, the expression levels of both $\operatorname{rsm} Y$ and $r s m Z$ are indeed negatively affected in the double $\triangle h p t B g a c A$ in a comparable way to the simple $\triangle g a c A$ mutant, supporting the hypothesis that the control of $\mathrm{HptB}$ over $r s m Y$ and $r s m Z$ occurs downstream of GacA (Figure 4A). Similarly, when the same experiment was performed on swarmer cells (surface-growing bacteria), a similar negative effect of $\mathrm{HptB}$ on $r s m Y$ was confirmed to be mediated through GacA. On the other hand, unexpectedly, the overexpression of $r s m Z$ that was seen in the $\Delta h p t B$ mutant background (Figure 2A) is still observed when $g a c A$ is also abrogated (Figure 4B), indicating that $\mathrm{HptB}$ regulates $r s m Z$ expression via an unidentified alternative regulatory pathway independent of GacA, specific to bacteria growing on a surface.

\section{DISCUSSION}

Swarming motility is a complex multicellular phenomenon that has been extensively investigated over the years. However, the underlying genetic regulatory pathways controlling that surfaceassociated type of motility still remain to be fully characterized 
A

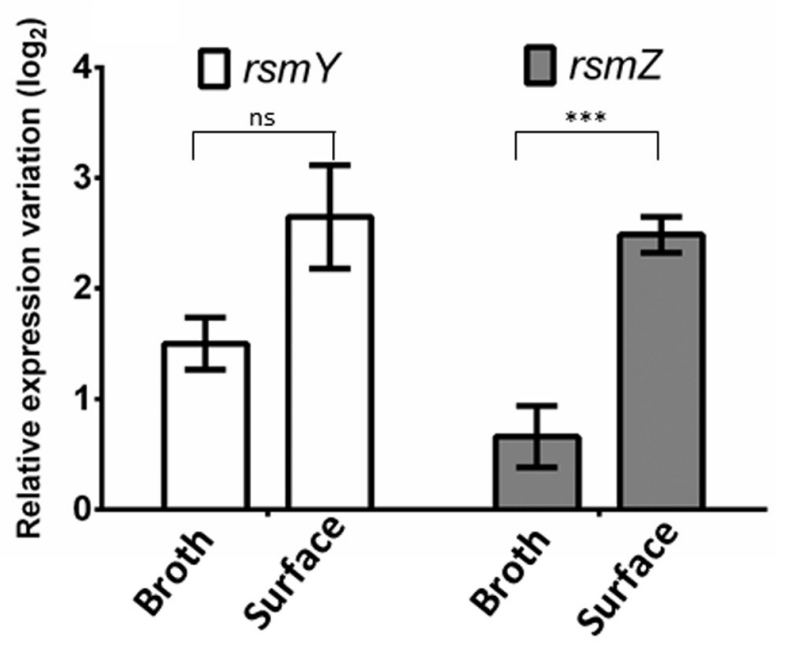

B

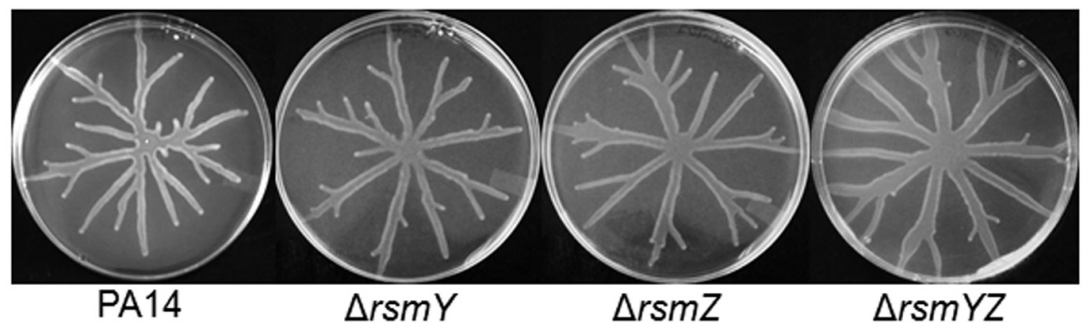

C

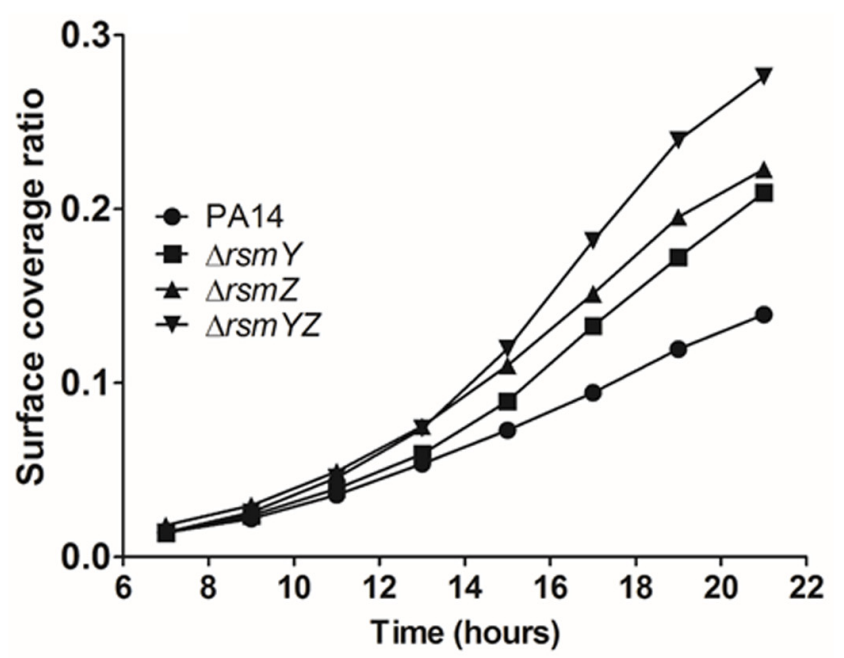

FIGURE 2 | Swarming motility is linked to sRNA expression. (A) Expression of the sRNAs RsmY and RsmZ in the $\triangle$ hptB mutant strain grown in broth or on a surface (swarming condition) determined by qRT-PCR. (B) Swarming motility of various sRNA mutants. (C) Time-lapse analysis of the $\Delta r s m Y / Z$ mutants. Error bars represent the standard error of the mean for experiments carried out at least twice with three biological replicates per experiment. Student's $t$-test analysis was applied on two independent experiments with ${ }^{* * *} p<0.001$; ns, not significant. For (C), the data correspond to one single plate per strain. Gene expression variation is shown as relative expression variation ( $\left.\log _{2}\right)$ to the wild-type PA14 strain.

in $P$. aeruginosa. In the present study, we have identified that the inactivation of the $h p t B$ gene renders $P$. aeruginosa incapable of such a type of motility even though this mutant still expresses the necessary propelling and wetting tools to exert a normal movement on a semi-solid ( $0.5 \%$ agar) medium.
The HptB protein has been well-described and novel pathways implicating this protein have been identified. Bhuwan et al. (2012), saw that transcription of many flagella-related genes was affected in a $\Delta h p t B$ mutant grown as swarming cells and that these effects were mediated via a novel regulatory 


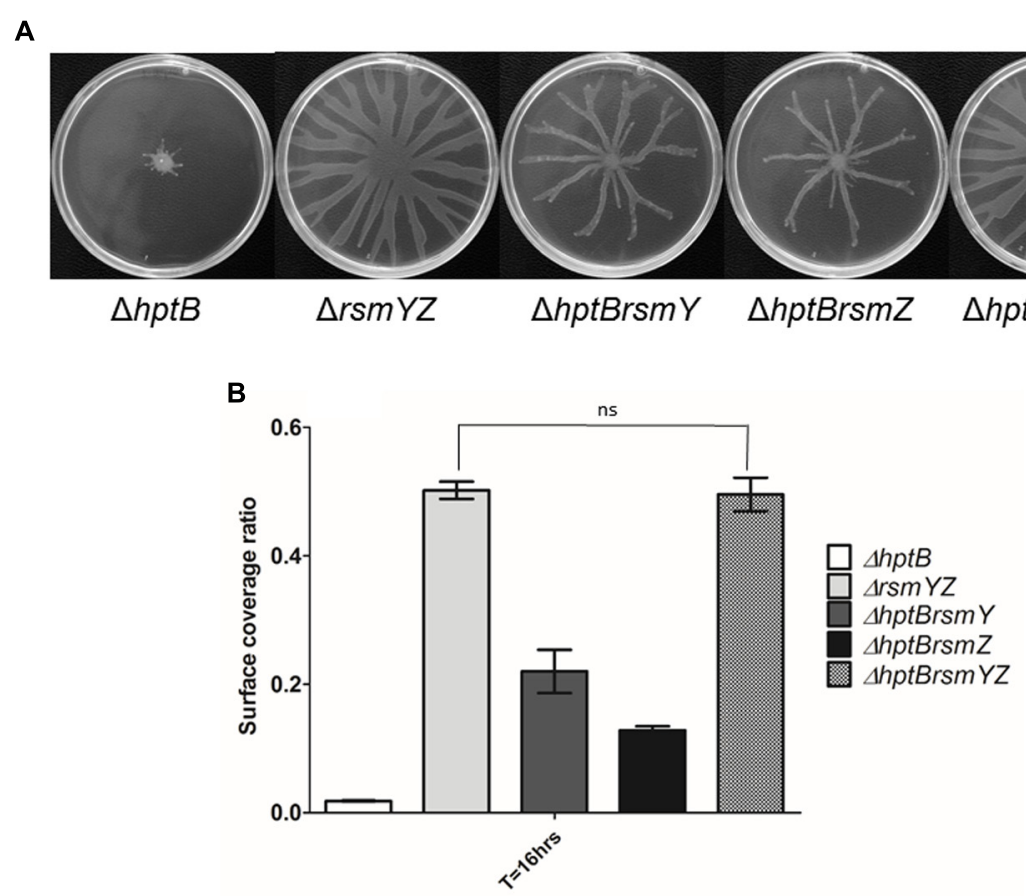

FIGURE 3 | Swarming deficiency of the $\mathbf{A} \boldsymbol{h p t B}$ mutant is due to sRNA overexpression. (A) Swarming motility assessment of various double and triple mutants. Shown are the average representative swarming phenotype of various strains. (B) Swarming motility surface coverage of the simple $\Delta h p t B$ mutant, the double $\Delta$ hptBrsmY/Z mutants, the triple $\Delta$ hptBrsm $Y Z$ mutant and $\Delta r s m Y Z$ double mutant. Student's $t$-test analysis was done on the $\Delta r s m Y Z$ double mutant and the triple $\Delta$ htBrsm YZ mutant based on two independent experiments (ns, not significant). Error bars represent the standard deviation of three technical replicates.

cascade implicating the PA3346 and PA3347 gene products. Surprisingly, they reported no differences in flagellar morphology and swimming motility of the $\Delta h p t B$ mutant, indicating that the functionality of this propelling appendage was apparently not affected. Thus, a regulatory imbalance in that mutant was possibly responsible for such a phenotype. However, Lin et al. (2006), observed that the absence of HptB provoked an important decrease in swimming motility in the PAO1 strain. In contrast to these studies, we looked at flagellar functionality and did not observe a defect in swimming motility of the $\Delta h p t B$ mutant that could explain the dramatic decrease in swarming motility. The discrepancies observed between these two studies and ours could be due to differences in experimental design and strains. Bhuwan et al. (2012), incubated their swarming plates at $30^{\circ} \mathrm{C}$ for $36 \mathrm{~h}$ before proceeding to their transcriptomic analyses. Furthermore, they looked at their swarming phenotypes by incubating their plates at $37^{\circ} \mathrm{C}$ for $36 \mathrm{~h}$. Our experiments were all carried out using plates incubated at $34^{\circ} \mathrm{C}$ for $12-16 \mathrm{~h}$, when the cells are still metabolically active (Tremblay and Déziel, 2010).

Also, previous studies looking at the implication of $\mathrm{HptB}$ in motility never addressed the question of RhlABC products (biosurfactants). To express the swarming phenotype, $P$. aeruginosa needs the production of the wetting agent rhamnolipids (Déziel et al., 2003; Caiazza et al., 2005). A $\Delta r h l A$ mutant is incapable of swarming motility thus looking at the production of the biosurfactants is imperative in studies investigating this type of surface-associated motility. Here, we looked at the production of rhamnolipids in the $\Delta h p t B$ mutant and did not see differences in production compared to the wild-type strain when the cells were cultivated in liquid cultures. Interestingly the same mutant produced more rhamnolipids than wild-type PA14 under swarming conditions. We hypothesize that rhamnolipid production is upregulated to overcome the absence of swarming. Accordingly, a 1:1 co-culture of the $\Delta h p t B$ and $r h l A^{-}$mutants results in a rescue of swarming motility of a $\triangle r h l A$ mutant strain (data not shown) and therefore we have no reason to believe that overproduction of these wetting agents would prevent such a type of motility, quite the opposite. Thus, $h p t B$ was considered an interesting gene to investigate how swarming motility is regulated.

The membrane sensor RetS is capable of phosphorylating the HptB protein (Hsu et al., 2008). Transcriptomic analyses performed on planktonic bacteria have revealed that the $\mathrm{HptB}$ and RetS regulons are partially overlapping but consist of two separate signaling pathways that both converge to the GacS/GacA system through different mechanisms (Bordi et al., 2010). Interestingly, HptB was seen to have an effect on the regulation of the small RNA RsmY specifically and implicated an alternative pathway including the PA3346 and PA3347 gene products whereas no effect of the phosphotransfer protein was observed on RsmZ (Bordi et al., 2010). Thus, since $\mathrm{HptB}$ seems to control many phenotypes via RsmY 


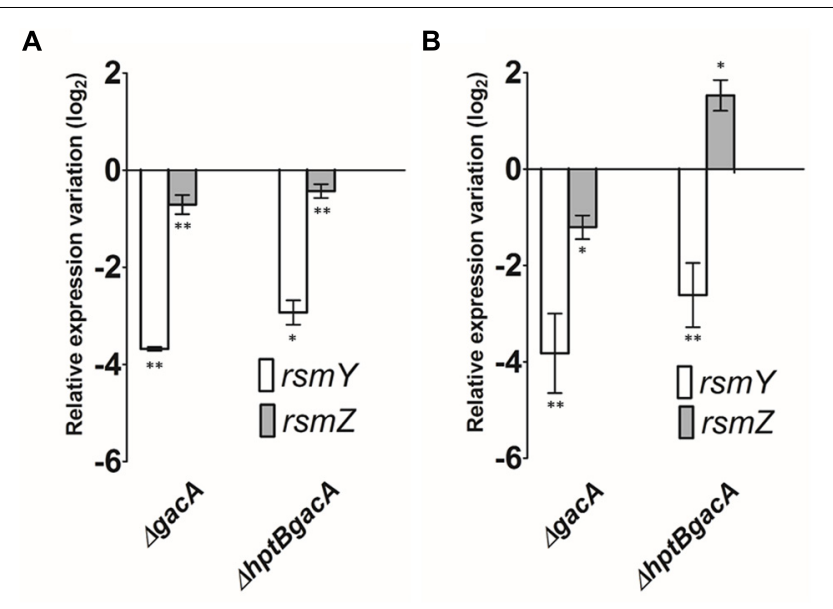

FIGURE 4 | The expression of sRNA is dependent of growth conditions. (A) Expression of rsmY and rsmZ determined by qRT-PCR in various genetic backgrounds grown in broth. (B) Expression of $r s m Y$ and $r s m Z$ of strains grown on a surface (swarming). Error bars represent the standard deviation of experiments carried out using three biological replicates. Student's $t$-test analysis was based on two independent experiments $\left({ }^{*} p<0.05 ;{ }^{* *} p<0.01\right)$. Gene expression variation is shown as relative expression variation $\left(\log _{2}\right)$ to the wild-type PA14 strain.

regulation we investigated the effect of a $\Delta h p t B$ mutation on small RNA regulation in both planktonic and swarming cells and confirmed the increased transcription of $\operatorname{rsm} Y$ in both conditions at the same extent. However, in contrast with that study, we observe a moderate increase in $r s m Z$ expression in a planktonic culture (Figure 2A). Since swarming is a surface-associated bacterial behavior, we also looked at the expression of both $r \sin Y$ and $r s m Z$ on cells that were collected at the tip of a migrating colony. Unexpectedly, we observed a $2 \log _{2}$ increase in expression of $r s m Z$ in swarming cells compared to their planktonic counterparts. This result indicates that $\operatorname{rsm} Z$ is differently regulated when cells are grown on a surface specifically. Such a different regulation on sRNAs is not unusual. For instance, Petrova and Sauer (2010) found that the inactivation of $b f i S$, implicated in biofilm formation, resulted in increased $r s m Y$ and $r s m Z$ expression strictly in cells cultivated as biofilms but not in planktonic ones. Here, we found only rsm $Z$ to be upregulated when comparing cells cultivated in broth versus a surface. This intriguing result guided us toward asking whether RsmZ could be the main mediator of swarming motility. Thus, we decided to look at the swarming phenotype of the simple and double $\Delta r s m Y / Z$ mutants. We expected to see an effect on swarming only for the $\Delta r s m Z$ mutant. Contrary to what has been observed by other groups (Heurlier et al., 2004; Kay et al., 2006), we saw an increase in swarming motility of both single mutants. The inactivation of both genes resulted in an even better capacity to swarm (Figures 2B,C). These results indicate that both RsmY and RsmZ act as negative regulatory elements of swarming motility and that the observed surface motility defect of the $\triangle h p t B$ mutant is explained by the overexpression of these two sRNA. As a matter of fact, we have also observed that the overexpression of either RsmY or RsmZ in the wild-type PA14 background results in a decrease in swarming motility (data not shown). Interestingly, other reporter phenotypes such as increased exopolysaccharide production and hyperbiofilm formation seen in the PAK $\Delta h p t B$ (Bordi et al., 2010) was not detected in a PA14 $\Delta$ hptB mutant (Supplementary Figures S3 and S4), indicating that these strains behave differently, as previously reported for swarming motility for instance (Tremblay and Déziel, 2008).

To associate without any further doubt the implication of $\mathrm{HptB}$ in the regulation of swarming motility via the downregulation of both $\operatorname{rsm} Y$ and $\operatorname{rsm} Z$, we created a triple $\triangle$ hptBrsmYZ mutant. Swarming motility assay of that mutant resulted in a complete rescue of the phenotype equivalent to that of the double $\triangle r s m Y Z$ strain. Swarming assessment of both double $\Delta h p t B r s m Y$ and $\Delta h p t B r s m Z$ mutants somehow resulted in an intermediate surface motility phenotype, further confirming that both sRNAs are important for this type of surface-associated movement and have a cumulative effect. Furthermore, Bordi et al. (2010) demonstrated a corresponding sRNA summative effect, as the abolishment of either $r s m Y$ or $r s m Z$ in a PAK $\Delta h p t B$ mutant background resulted in the production of intermediate biofilm phenotypes, whereas a triple $\triangle h p t B r s m Y Z$ mutant strain behaved exactly like a double $\triangle r s m Y Z$ mutant. Together, these findings validate that the investigated phenotypes affected by the deletion of the $h p t B$ gene (in PA14 and PAK strains) are linked to $\operatorname{rsm} Y$ and $\operatorname{rsm} Z$ overexpression.

Interestingly, an $r s m A^{-}$mutant is not as defective in swarming motility as the $\triangle h p t B$ mutant (Supplementary Figure S5). Also, the inactivation of $r s m A$ leads to impairment of rhamnolipids synthesis thus explaining its incapacity to swarm properly (Heurlier et al., 2004) while it is not the case for the $\Delta h p t B$ mutant. Recently, the novel post-transcriptional regulator, $\mathrm{RsmN}$ (an RsmA ortholog) has been described as a positive regulator of swarming motility (Morris et al., 2013). However, it was shown that the mutation of the $\operatorname{rsm} N$ gene did not abolish swarming motility, but rather decreased it. Investigating the effect of a double $r s m A r s m N$ mutant on swarming motility remains to be further studied. However, since the only known major target of both $\operatorname{rsm} Y$ and $r s m Z$ is the RsmA posttranscriptional repressor (Brencic and Lory, 2009), our data strongly suggest that these sRNA have alternative targets, yet to be identified.

As there is increasing evidence that sRNA control by HptB can be due to the alternative PA3346/PA3347 regulation pathway and to understand how that phosphotransfer protein can have an effect of sRNA regulation, we created a double $\triangle h p t B g a c A$ mutant. Knowing that GacA is the main positive regulator of rsm $Y$ and rsm $Z$ expression (Brencic et al., 2009), we expected to see a loss of sRNA upregulation in that double mutant. As anticipated, we observed a downregulation of both $r s m Y$ and $r s m Z$ in a planktonic culture of the $\triangle g a c A$ mutant as it has already been reported by other groups (Kay et al., 2006; Brencic and Lory, 2009) as well as in swarming cells of that same genotype (Figure 4). Furthermore, the introduction of an $h p t B$ deletion in the $\triangle$ gacA mutant strain resulted in the abolishment of both 


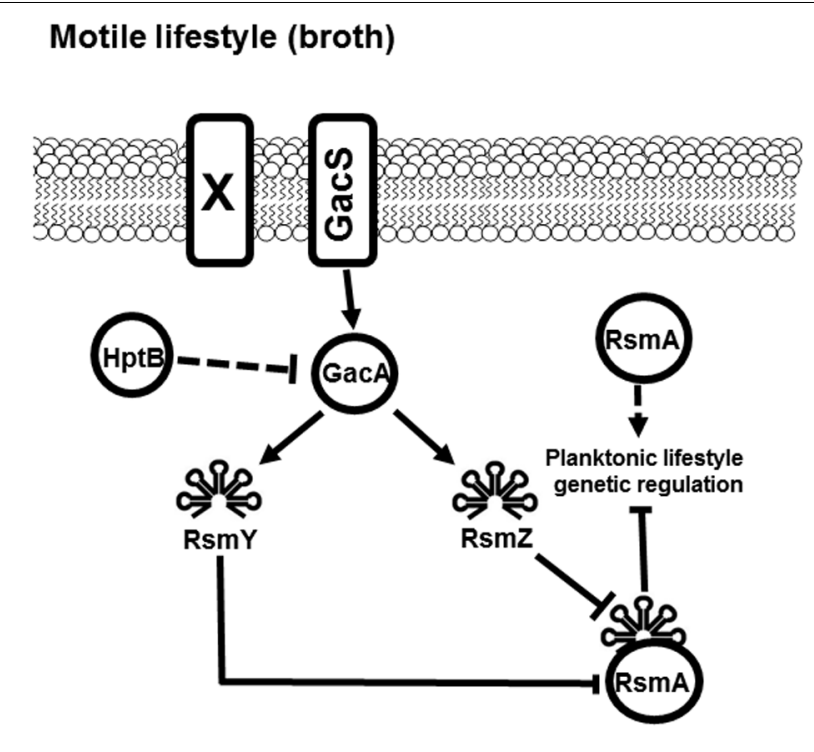

\section{Surface lifestyle (swarming)}

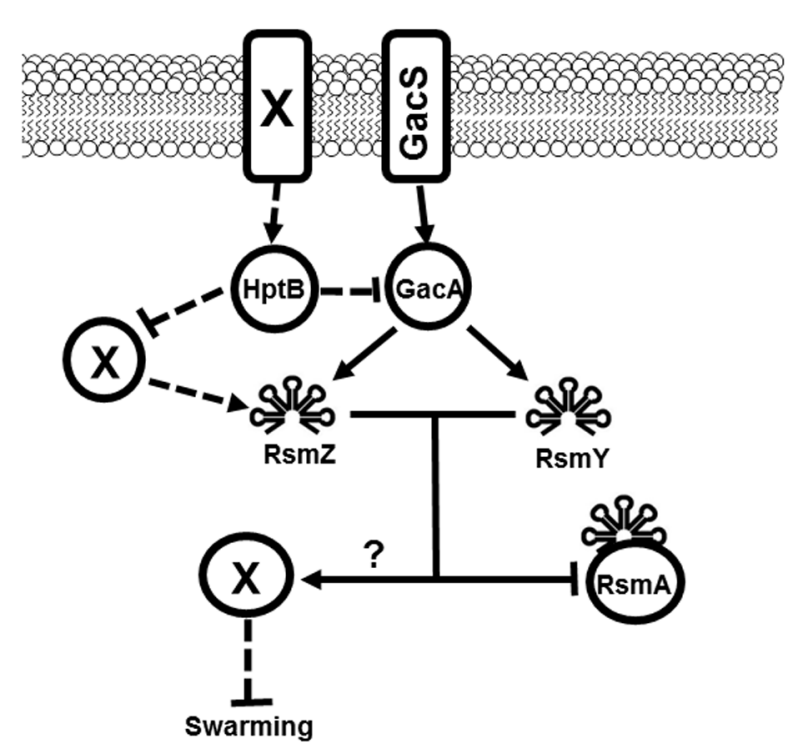

FIGURE 5 | Model for broth-surface differential sRNA regulation. We propose a regulation model where the control of the expression of $r s m Y$ and $r s m Z$ by $\mathrm{HptB}$ is under the exclusive control of the GacS/GacA system and converges to the post-transcriptional regulator RsmA for both planktonic and swarming cells. However, in cells grown on a surface such as in swarming motility, the regulation of both rsmY and rsmZ is differential and does not strictly mediate their output via RsmA. The obtained data in our study indicates that other key-players allows for regulation of $r s m Z$ by HptB and does not implicate the Gac system. This regulation most likely involves other membrane sensors that can modulate the activity of the HptB protein. Presented model integrates previously published data (Hsu et al., 2008; Brencic et al., 2009; Bordi et al., 2010). Full arrows represent direct positive regulation. Dashed arrows represent indirect positive regulation. Dashed bars represent indirect negative control. Full bars represent direct negative regulation. ? = unknown contribution. $\mathrm{X}=$ Unknown regulating factor.

rsm $Y$ and $r s m Z$ upregulation that was observed in the simple $\Delta h p t B$ mutant in liquid cultures. Interestingly, Bordi et al. (2010) observed that the hyperbiofilm phenotype of a PAK $\Delta h p t B$ strain was indeed abolished in a double $\triangle h p t B g a c A$ mutant suggesting that both sRNAs are implicated in the control of such a phenotype in strain PAK. Surprisingly in swarming cells, we observed that $\operatorname{rsm} Z$ was still upregulated when compared to a simple $\triangle$ gacA mutant whereas rsm $Y$ overexpression was lost in a double $\Delta h p t B g a c A$ mutant. This result reveals a differential regulation of both sRNAs in swarming cells, where rsm $Z$ transcription does not require the presence of $\mathrm{GacA}$ as it has been seen for planktonic cultures, but rather necessitates the contribution of unknown regulator(s) that are specifically active in surface-grown cells.

In addition of GacA being the main activator of $\operatorname{rsm} Y$ and $r s m Z$ transcription (Brencic et al., 2009), there are other global regulators that can influence the expression levels of these sRNAs in planktonic cultures. Accordingly, the DNA-binding global negative regulators $\mathrm{MvaT}$ and $\mathrm{MvaU}$, can specifically repress rsmZ expression without affecting rsmY (Castang et al., 2008) thus possibly being the reason why the upregulation was observed in surface-grown cells. We assessed the swarming motility of both $\Delta m v a T$ and $\Delta m v a U$ mutant strains and did not observe any difference in surface movement when compared to the wild-type (Supplementary Figure S6), indicating that any effect on $r s m Z$ regulation does not strictly exert an output on swarming motility.

To rule out the possibility of sRNA regulation by the PA3346/PA3347 pathway, we monitored the expression of rsm $Y$ and $r s m Z$ in both swarming cells and their planktonic counterpart of mutants in these genes. Opposite to Bordi et al. (2010), we observed a slight increase in $\operatorname{rsm} Y$ expression in the $P_{3346^{-}}$mutant background but no effect for both sRNAs in $P A 3347^{-}$in planktonic cultures (Supplementary Figure S7). Also, the inactivation of either PA3346 or PA3347 did not affect rsmY and $r s m Z$ expression in surface-grown cells (Supplementary Figure S8). These results indicated us that $r s m Z$ overexpression in a $\triangle h p t B$ genetic mutant background is somehow independent of both GacS/GacA and PA3346/47 regulation pathways and that an unknown surface-activated regulator is responsible for the observed effect. Recently, a study by Wang et al. (2014), characterized a novel swarming regulator, BswR, capable of controlling the expression of $r \sin Z$ directly in PAO1. We verified whether BswR could be responsible for $r s m Z$ regulation in swarming cells, but, surprisingly, we did not observe any effect on $r s m Z$ expression in a $\Delta b s w R$ mutant in both planktonic and swarming cells (Supplementary Figure S9), indicating that BswR does not play a role in the regulation of this sRNA under our conditions. Earlier this year, a study published by Xu et al. (2016) characterized the HapZ adaptor protein in a PAO1 strain and observed that it could act as an intermediate between the membrane sensor SagS and the HptB protein (Xu et al., 2016). However, it was reported that a mutation in the sagS gene does not affect swarming motility in PA14 (Petrova and Sauer, 2011), making HapZ unlikely to participate in swarming motility regulation, at least, in a PA14 genetic background. 
The discrepancies observed between our study and other ones (Bordi et al., 2010; Bhuwan et al., 2012; Wang et al., 2014) stress out the growing evidence that there are regulatory differences between the various $P$. aeruginosa strains (Tremblay and Déziel, 2008; Mikkelsen et al., 2011). Furthermore, we certainly cannot rule out the implication of the numerous previously characterized elements found to be implicated in the regulation of swarming motility. However, here we present a model where small RNA regulation is dependent on the conditions in which we study $P$. aeruginosa (i.e., broth versus surface) which favor two different bacterial lifestyles. Furthermore, RsmW a novel RNA under the control of the Gac system has been identified to be strongly upregulated in biofilm conditions compared to planktonic cultures which further strengthens the existence of differential genetic regulation depending on the selected growth conditions (Miller et al., 2016). Our data confirm that the GacS/GacA system is not the only one responsible for the control of $r \sin Z$ expression and implicates the presence of unidentified key factors that are important when cells are grown on a surface rather than in planktonic cultures, as exemplified by the use of swarming motility as a model for surface behavior (Figure 5). Further downstream in the swarming regulatory cascade, we also show evidence that RsmY and RsmZ can modulate swarming motility not only via RsmA inhibition. Further experiments focusing on identifying these regulatory players specific to surface-grown bacteria will unveil a whole misunderstood genetic regulation portrait of P. aeruginosa.

\section{REFERENCES}

Abdel-Mawgoud, A., Lépine, F., and Déziel, E. (2014). "Liquid chromatography/mass spectrometry for the identification and quantification of rhamnolipids," in Pseudomonas Methods and Protocols, eds A. Filloux and J.-L. Ramos (New York, NY: Springer), 359-373.

Baker, A. E., Diepold, A., Kuchma, S. L., Scott, J. E., Ha, D. G., Orazi, G., et al. (2016). PilZ domain protein FlgZ mediates cyclic Di-GMP-dependent swarming motility control in Pseudomonas aeruginosa. J. Bacteriol. 198, 18371846. doi: 10.1128/JB.00196-16

Beier, D., and Gross, R. (2006). Regulation of bacterial virulence by twocomponent systems. Curr. Opin. Microbiol. 9, 143-152. doi: 10.1016/j.mib.2006. 01.005

Bhuwan, M., Lee, H. J., Peng, H. L., and Chang, H. Y. (2012). Histidinecontaining phosphotransfer protein-B (HptB) regulates swarming motility through partner-switching system in Pseudomonas aeruginosa PAO1 strain. J. Biol. Chem. 287, 1903-1914. doi: 10.1074/jbc.M111.256586

Boor, K. J. (2006). Bacterial stress responses: what doesn't kill them can make them stronger. PLoS Biol. 4:e23. doi: 10.1371/journal.pbio.0040023

Bordi, C., Lamy, M. C., Ventre, I., Termine, E., Hachani, A., Fillet, S., et al. (2010). Regulatory RNAs and the HptB/RetS signalling pathways fine-tune Pseudomonas aeruginosa pathogenesis. Mol. Microbiol. 76, 1427-1443. doi: 10. 1111/j.1365-2958.2010.07146.x

Brencic, A., and Lory, S. (2009). Determination of the regulon and identification of novel mRNA targets of Pseudomonas aeruginosa RsmA. Mol. Microbiol. 72, 612-632. doi: 10.1111/j.1365-2958.2009.06670.x

Brencic, A., McFarland, K. A., McManus, H. R., Castang, S., Mogno, I., Dove, S. L., et al. (2009). The GacS/GacA signal transduction system of Pseudomonas aeruginosa acts exclusively through its control over the transcription of the RsmY and RsmZ regulatory small RNAs. Mol. Microbiol. 73, 434-445. doi: 10.1111/j.1365-2958.2009.06782.x

\section{AUTHOR CONTRIBUTIONS}

FJ-P, JT, and ED conceived and designed the experiments. FJ-P and JT performed the experiments. FJ-P, JT, and ED analyzed the data. FJ-P, JT, and ED contributed to manuscript preparation and editing.

\section{ACKNOWLEDGMENTS}

We thank Dr. Steve Lory for providing the pEXG2$\Delta r s m Y / p E X G 2-\Delta r s m Z$ suicide plasmids and the pCTX-rsm $Y$ lacZ/pCTX-rsmZ-lacZ reporters, Marie-Christine Groleau for helpful discussions and Sophie Robitaille for technical help. This study was supported by Natural Sciences and Engineering Research Council of Canada (NSERC) Discovery grant RGPIN2015-03931 awarded to ED. ED holds the Canada Research Chair in Sociomicrobiology. FJ-P was recipient of Ph.D. scholarships from the Fonds de Recherche - Nature et Technologies (FRQNT) and the Fondation Universitaire Armand-Frappier de l'INRS.

\section{SUPPLEMENTARY MATERIAL}

The Supplementary Material for this article can be found online at: http://journal.frontiersin.org/article/10.3389/fmicb. 2016.02168/full\#supplementary-material

Caiazza, N. C., Merritt, J. H., Brothers, K. M., and O'Toole, G. A. (2007). Inverse regulation of biofilm formation and swarming motility by Pseudomonas aeruginosa PA14. J. Bacteriol. 189, 3603-3612. doi: 10.1128/JB. 01685-06

Caiazza, N. C., Shanks, R. M., and O’Toole, G. A. (2005). Rhamnolipids modulate swarming motility patterns of Pseudomonas aeruginosa. J. Bacteriol. 187, 73517361. doi: 10.1128/JB.187.21.7351-7361.2005

Castang, S., McManus, H. R., Turner, K. H., and Dove, S. L. (2008). H-NS family members function coordinately in an opportunistic pathogen. Proc. Natl. Acad. Sci. U.S.A. 105, 18947-18952. doi: 10.1073/pnas.0808215105

Déziel, E., Lépine, F., Milot, S., and Villemur, R. (2003). rhlA is required for the production of a novel biosurfactant promoting swarming motility in Pseudomonas aeruginosa: 3-(3-hydroxyalkanoyloxy)alkanoic acids (HAAs), the precursors of rhamnolipids. Microbiology 149(Pt 8), 2005-2013. doi: 10.1099/ mic.0.26154-0

Furukawa, S., Kuchma, S. L., and O’Toole, G. A. (2006). Keeping their options open: acute versus persistent infections. J. Bacteriol. 188, 1211-1217. doi: 10. 1128/JB.188.4.1211-1217.2006

Goodman, A. L., Kulasekara, B., Rietsch, A., Boyd, D., Smith, R. S., and Lory, S. (2004). A signaling network reciprocally regulates genes associated with acute infection and chronic persistence in Pseudomonas aeruginosa. Dev. Cell 7, 745-754. doi: 10.1016/j.devcel.2004.08.020

Heurlier, K., Williams, F., Heeb, S., Dormond, C., Pessi, G., Singer, D., et al. (2004). Positive control of swarming, rhamnolipid synthesis, and lipase production by the posttranscriptional RsmA/RsmZ system in Pseudomonas aeruginosa PAO1. J. Bacteriol. 186, 2936-2945. doi: 10.1128/JB.186.10.2936-2945.2004

Hoang, T. T., Karkhoff-Schweizer, R. R., Kutchma, A. J., and Schweizer, H. P. (1998). A broad-host-range Flp-FRT recombination system for site-specific excision of chromosomally-located DNA sequences: application for isolation of unmarked Pseudomonas aeruginosa mutants. Gene 212, 77-86. doi: 10.1016/ S0378-1119(98)00130-9 
Hoch, J. A., and Varughese, K. I. (2001). Keeping signals straight in phosphorelay signal transduction. J. Bacteriol. 183, 4941-4949. doi: 10.1128/jb.183.17.49414949.2001

Hsu, J. L., Chen, H. C., Peng, H. L., and Chang, H. Y. (2008). Characterization of the histidine-containing phosphotransfer protein B-mediated multistep phosphorelay system in Pseudomonas aeruginosa PAO1. J. Biol. Chem. 283, 9933-9944. doi: 10.1074/jbc.M708836200

Kay, E., Humair, B., Denervaud, V., Riedel, K., Spahr, S., Eberl, L., et al. (2006). Two GacA-dependent small RNAs modulate the quorum-sensing response in Pseudomonas aeruginosa. J. Bacteriol. 188, 6026-6033. doi: 10.1128/JB. 00409-06

Kearns, D. B. (2010). A field guide to bacterial swarming motility. Nat. Rev. Microbiol. 8, 634-644. doi: 10.1038/nrmicro2405

Köhler, T., Curty, L. K., Barja, F., van Delden, C., and Pechere, J. C. (2000). Swarming of Pseudomonas aeruginosa is dependent on cell-to-cell signaling and requires flagella and pili. J. Bacteriol. 182, 5990-5996. doi: 10.1128/JB.182.21. 5990-5996.2000

Kong, W., Chen, L., Zhao, J., Shen, T., Surette, M. G., Shen, L., et al. (2013). Hybrid sensor kinase PA1611 in Pseudomonas aeruginosa regulates transitions between acute and chronic infection through direct interaction with RetS. Mol. Microbiol. 88, 784-797. doi: 10.1111/mmi.12223

Kuchma, S. L., Brothers, K. M., Merritt, J. H., Liberati, N. T., Ausubel, F. M., and O’Toole, G. A. (2007). BifA, a cyclic-Di-GMP phosphodiesterase, inversely regulates biofilm formation and swarming motility by Pseudomonas aeruginosa PA14. J. Bacteriol. 189, 8165-8178. doi: 10.1128/JB.00586-07

Lai, S., Tremblay, J., and Déziel, E. (2009). Swarming motility: a multicellular behaviour conferring antimicrobial resistance. Environ. Microbiol. 11, 126-136. doi: 10.1111/j.1462-2920.2008.01747.x

Lee, D. G., Urbach, J. M., Wu, G., Liberati, N. T., Feinbaum, R. L., Miyata, S., et al. (2006). Genomic analysis reveals that Pseudomonas aeruginosa virulence is combinatorial. Genome Biol. 7, 1-14. doi: 10.1186/gb-2006-7-10-r90

Liberati, N. T., Urbach, J. M., Miyata, S., Lee, D. G., Drenkard, E., Wu, G., et al. (2006). An ordered, nonredundant library of Pseudomonas aeruginosa strain PA14 transposon insertion mutants. Proc. Natl. Acad. Sci. U.S.A. 103, 2833-2838. doi: 10.1073/pnas.0511100103

Lin, C. T., Huang, Y. J., Chu, P. H., Hsu, J. L., Huang, C. H., and Peng, H. L. (2006). Identification of an HptB-mediated multi-step phosphorelay in Pseudomonas aeruginosa PAO1. Res. Microbiol. 157, 169-175. doi: 10.1016/j.resmic.2005.06. 012

Livak, K. J., and Schmittgen, T. D. (2001). Analysis of relative gene expression data using real-time quantitative PCR and the 2(-Delta Delta C(T)) Method. Methods 25, 402-408. doi: 10.1006/meth.2001.1262

Merritt, J. H., Brothers, K. M., Kuchma, S. L., and O’Toole, G. A. (2007). SadC reciprocally influences biofilm formation and swarming motility via modulation of exopolysaccharide production and flagellar function. J. Bacteriol. 189, 8154-8164. doi: 10.1128/JB.00585-07

Mikkelsen, H., McMullan, R., and Filloux, A. (2011). The Pseudomonas aeruginosa reference strain PA14 displays increased virulence due to a mutation in ladS. PLoS ONE 6:e29113. doi: 10.1371/journal.pone.0029113

Miller, C. L., Romero, M., Karna, S. L., Chen, T., Heeb, S., and Leung, K. P. (2016). RsmW, Pseudomonas aeruginosa small non-coding RsmA-binding RNA upregulated in biofilm versus planktonic growth conditions. BMC Microbiol. 16:155. doi: 10.1186/s12866-016-0771-y

Morris, E. R., Hall, G., Li, C., Heeb, S., Kulkarni, R. V., Lovelock, L., et al. (2013). Structural rearrangement in an RsmA/CsrA Ortholog of Pseudomonas aeruginosa creates a dimeric RNA-binding protein, RsmN. Structure 21, 16591671. doi: 10.1016/j.str.2013.07.007

Overhage, J., Bains, M., Brazas, M. D., and Hancock, R. E. (2008). Swarming of Pseudomonas aeruginosa is a complex adaptation leading to increased production of virulence factors and antibiotic resistance. J. Bacteriol. 190, 2671-2679. doi: 10.1128/JB.01659-07

Petrova, O. E., and Sauer, K. (2010). The novel two-component regulatory system BfiSR regulates biofilm development by controlling the small RNA rsmZ through CafA. J. Bacteriol. 192, 5275-5288. doi: 10.1128/JB. 00387-10

Petrova, O. E., and Sauer, K. (2011). SagS contributes to the motile-sessile switch and acts in concert with BfiSR to enable Pseudomonas aeruginosa biofilm formation. J. Bacteriol. 193, 6614-6628. doi: 10.1128/JB.00305-11

Rahme, L. G., Stevens, E. J., Wolfort, S. F., Shao, J., Tompkins, R. G., and Ausubel, F. M. (1995). Common virulence factors for bacterial pathogenicity in plants and animals. Science 268, 1899-1902. doi: 10.1126/science.7604262

Rodrigue, A., Quentin, Y., Lazdunski, A., Mejean, V., and Foglino, M. (2000). Two-component systems in Pseudomonas aeruginosa: why so many? Trends Microbiol. 8, 498-504. doi: 10.1016/S0966-842X(00)01833-3

Simon, R., Priefer, U., and Puhler, A. (1983). A broad host range mobilization system for in vivo genetic engineering: transposon mutagenesis in gram negative bacteria. Nat. Biotechnol. 1, 784-791. doi: 10.1038/nbt1183-784

Tremblay, J., and Déziel, E. (2008). Improving the reproducibility of Pseudomonas aeruginosa swarming motility assays. J. Basic Microbiol. 48, 509-515. doi: 10. 1002/jobm.200800030

Tremblay, J., and Déziel, E. (2010). Gene expression in Pseudomonas aeruginosa swarming motility. BMC Genomics 11:587. doi: 10.1186/1471-2164-11-587

Tremblay, J., Richardson, A. P., Lepine, F., and Déziel, E. (2007). Self-produced extracellular stimuli modulate the Pseudomonas aeruginosa swarming motility behaviour. Environ. Microbiol. 9, 2622-2630. doi: 10.1111/j.1462-2920.2007. 01396.x

Wang, C., Ye, F., Kumar, V., Gao, Y.-G., and Zhang, L.-H. (2014). BswR controls bacterial motility and biofilm formation in Pseudomonas aeruginosa through modulation of the small RNA rsmZ. Nucleic Acids Res. 42, 4563-4576. doi: 10.1093/nar/gku106

Woodcock, D. M., Crowther, P. J., Doherty, J., Jefferson, S., DeCruz, E., Noyer-Weidner, M., et al. (1989). Quantitative evaluation of Escherichia coli host strains for tolerance to cytosine methylation in plasmid and phage recombinants. Nucleic Acids Res. 17, 3469-3478. doi: 10.1093/nar/17. 9.3469

Xu, L., Venkataramani, P., Ding, Y., Liu, Y., Deng, Y., Yong, G. L., et al. (2016). A Cyclic di-GMP-binding adaptor protein interacts with histidine kinase to regulate two-component signaling. J. Biol. Chem. 291, 16112-16123. doi: 10. 1074/jbc.M116.730887

Conflict of Interest Statement: The authors declare that the research was conducted in the absence of any commercial or financial relationships that could be construed as a potential conflict of interest.

Copyright (c) 2017 Jean-Pierre, Tremblay and Déziel. This is an open-access article distributed under the terms of the Creative Commons Attribution License (CC BY). The use, distribution or reproduction in other forums is permitted, provided the original author(s) or licensor are credited and that the original publication in this journal is cited, in accordance with accepted academic practice. No use, distribution or reproduction is permitted which does not comply with these terms. 